\title{
Extrinsic Risk Factors for Women Breast Cancer in Gaza Strip, Palestine: Associations and Interactions in a Case-Control Study
}

\author{
Samir Yassin ${ }^{1}$, Malak Younis ${ }^{1}$, Samer Abuzerr ${ }^{2,3^{*}}$, Maher Darwish ${ }^{4}$, Ayman Abu Mustafa $^{5}$ \\ ${ }^{1}$ Department of Physics, Faculty of Sciences, Islamic University of Gaza, Gaza, Palestine \\ ${ }^{2}$ Department of Environmental Health Engineering, Faculty of Public Health, International Campus, Tehran University of \\ Medical Sciences, Tehran, Iran \\ ${ }^{3}$ Unit of Quality Improvement and Infection Control, Ministry of Health, Gaza, Palestine \\ ${ }^{4}$ Department of Pharmacy, Al-Safwa University College, Karbala, Iraq \\ ${ }^{5}$ Department of Research, Directorate General of Human Resources Development, Ministry of Health, Gaza, Palestine \\ Email: *samer_516@hotmail.com, syassin@iugaza.edu.ps, myounis@iugaza.edu.ps,m-darwish@razi.tums.ac.ir, \\ aymanayman20092009@hotmail.com
}

How to cite this paper: Yassin, S., Younis, M., Abuzerr, S., Darwish, M. and Mustafa, A.A. (2019) Extrinsic Risk Factors for Women Breast Cancer in Gaza Strip, Palestine: Associations and Interactions in a Case-Control Study. Advances in Breast Cancer Research, 8, 11-30.

https://doi.org/10.4236/abcr.2019.81002

Received: November 15, 2018

Accepted: December 22, 2018

Published: December 25, 2018

Copyright (C) 2019 by authors and Scientific Research Publishing Inc. This work is licensed under the Creative Commons Attribution International License (CC BY 4.0).

http://creativecommons.org/licenses/by/4.0/

\begin{abstract}
Background: Worldwide, breast cancer $(\mathrm{BC})$ is the most frequently diagnosed neoplastic disease in women around menopause that is the leading cause of DALYs, because it causes a significant reduction of these women's ability to function normally in everyday life. Methods: The present hospital-based case-control study was carried out between January and August 2018 using a structured questionnaire on 105 breast cancer women and 210 controls who are clinically free from breast cancer. Data about the study cases were collected in the oncology day-care clinics of the two main hospitals in Gaza strip "Al-Shifa and European Gaza hospitals". Univariate logistic regression and multivariate logistic regression analyses were employed to identify the significant factors associated with BC. Results: Multivariate logistic regression analyses with adjustment for all confounders revealed that woman with BMI more than or equal $30 \mathrm{~kg} / \mathrm{m}^{2}$ are under risk of getting BC 2.9 times greater than those having BMI less than $29 \mathrm{~kg} / \mathrm{m}^{2}$ (AOR $=2.895 ; 95 \% \mathrm{CI}$ : $1.305-6.423)$. Analysis of risk according to reaching menopause showed that the estimated AOR was greater among those reached menopause (3.137, 95\% CI 1.824 - 5.395) than among those that did not reach menopause. The risk of developing $\mathrm{BC}$ in the case of a history of incidence of $\mathrm{BC}$ in the family was more than two times higher $(\mathrm{AOR}=2.632,95 \% \mathrm{CI} 1.528-4.535)$ than in case of the history of a free family of $\mathrm{BC}$. Conclusion: In this context, the above-mentioned risk factors must be taken into consideration in BC management processes in the Gaza strip.
\end{abstract}




\section{Keywords}

Breast Cancer, Case-Control, Risk Factors, Women, Gaza Strip

\section{Introduction}

Worldwide, breast cancer (BC) is the most common malignancy in women and the leading cause of cancer death. It is estimated that 1.5 million new cases of $\mathrm{BC}$ diagnosed annually with odds of getting BC is one in eight women [1] [2] [3]. Generally, high body mass index (BMI), low fruit and vegetable intake, lack of physical activity, tobacco use, and alcohol abuse are the five behavioral and dietary risks leading cause of $30 \%$ of cancer deaths [4] [5] [6]. International variation in female $\mathrm{BC}$ incidence and mortality rate could be attributed to differences in sociodemographic factors, reproductive patterns, lifestyle, and other hormonal factors [4] [7] [8]. Moreover, family history and age of women are the most two individual critical BC risk factors [9] [10].

In low-income countries, $\mathrm{BC}$ cases tend to young women with median age of 49 - 52 years whereas 63 years in high-income countries [11]. Early detection of $\mathrm{BC}$ would improve treatment and recovery, limit the complications and reduce the risk of mortality [12] [13] [14]. High BC mortality rate, particularly in developing countries, is due primarily to the detection of late-stage BC [15] [16]. Therefore, the American Cancer Society recommended that women should have the opportunity to begin annual $\mathrm{BC}$ screening around the age of 40 years and should be encouraged to discuss their family history and medical history with a clinician. Also, they should be provided with information on BC risk factors and risk reduction [17].

According to the Palestinian ministry of health $(\mathrm{MOH}), \mathrm{BC}$ is $31.3 \%$ of women's cancers in Gaza Strip and it is the second leading cause of female death after cardiovascular disease. Since the total number of reported BC cases between 2009 and 2014 was 1283 cases, out of them, 1207 were females and 76 were males. Furthermore, as has been estimated, the incidence rate of BC among Gaza's females was about 83.9 per 100,000 women between 2009 and 2014, which was increased to 154.2 per 100,000 women in 2015 [18].

To the best of our knowledge, there was limited documented research on the extrinsic risk factors for women $\mathrm{BC}$ in Gaza strip. Therefore, the objective of this case-control study was to identify the distinct associations between extrinsic risk factors and developing BC among women in the Gaza strip. Understanding such associations is important to further our understanding of disease etiology and to provide personalized prevention and treatment measures.

\section{Materials and Methods}

\subsection{Study Design and Setting}

The present hospital-based case-control study was conducted between January 
and August 2018 with a representative sample of BC women. The study sample was compromised of 105 BC patients and 210 controls. Data about the study cases were collected in the only two oncology day-care clinics in the two main hospitals in Gaza strip "Al-Shifa hospital in Gaza governorate and European Gaza hospitals in Khanyunis governorate".

\subsection{Study Tool and Sampling}

In this study, patients and controls were interviewed by well-trained interviewers. The interviewers used a structured and a validated questionnaire to collect information on sociodemographic characteristics and potential risk factors, such history of exposure to toxic gases, radiation and pesticides, body anthropometric measurements, history of medical conditions, history of maternal and reproductive health, lifestyle, and dietary habits.The computer program Epi Info version $6.04 \mathrm{~d}$ was used to calculate the sample size using $5 \%$ precision with $95 \%$ confidence interval from the study population comprised of 1207 breast cancer women to be 105 patients.

Patients and controls were questioned about age at breast cancer diagnosis and history of cancer and breast cancer among their first and second-degree relatives. No proxy interviews were conducted. Smokers were defined as subjects who had smoked $\geq 100$ cigarettes during their lifetime. Obesity measured by body mass index (BMI), a participant's weight (in kilograms) divided by the square of her height (in meters). A participant with a BMI of $30 \mathrm{~kg} / \mathrm{m}^{2}$ or more is considered obese. The dietary habits in our study contain a list of the 19 most common food groups; based on the results of the previous study [19]. Also, Participants were asked about the number of daily meals.

\subsection{Eligibility Criteria}

The case subjects were confirmed $\mathrm{BC}$ women by a histopathology test and recorded in one of the two above mentioned oncology day-care clinics. Whereas, the control subjects were healthy women and genetically unrelated family members of $\mathrm{BC}$ patients. The reason behind excluding family members of $\mathrm{BC}$ patients, as controls, is to avoid induction of selection bias. Control subjects were recruited from the vaccination departments at primary health care clinics, where women take their children for vaccination. Controls and patients were recruited simultaneously. The cases and controls were frequency matched by age group, marital status, governorate of residence, and nature of housing area.

\subsection{Ethical Consideration}

Approval to conduct the study was gotten from the Palestinian ministry of health. Also, written consent from participants was obtained after explaining the aim of the study. Great care was taken to ensure privacy and confidentiality.

\subsection{Data Analysis}

Data were analyzed using the Statistical Package for Social Science (SPSS) ver- 
sion 22 (IBM Corp, Armonk, NY, USA). Frequencies and percentages were calculated for all variables. Odds ratio (OR) with a 95\% confidence interval was used to investigate the strength of association between the determinant factors and the outcome variable. Univariate logistic regression analysis was used to identify risk factors associated with the incidence of $\mathrm{BC}$ at $\mathrm{p}<0.05$ and $95 \% \mathrm{CI}$ without controlling confounders. Multivariable logistic regression analysis was employed to examine the association between risk factors and BC incidence under controlling of potential confounding factors.

\section{Results}

\subsection{Sociodemographic Characteristics and Their Association with Breast Cancer}

As shown in Table 1, a total of 105 BC cases and 210 controls were enrolled in this study. In both cases and controls Gaza Governorate had the largest number of cases ( $n=48,45.7 \%$ ) as well as controls ( $n=96,45.7 \%)$. Age groups were divided into five groups since the age of the majority of the study participants was above 40 years (80\%). Eighty-one $(77.1 \%)$ of the cases were married. Regarding the educational level of the cases, $22(21 \%)$ hold a university degree and 19 (18.1\%) had a primary degree. Most of cases and controls, $(n=81,77.1 \%)$ and $(\mathrm{n}=162,77.1 \%)$, respectively were married. Approximately 81 breast cancer women (52.4\%) were diagnosed with BC at the age between 30 and 40 years, (n $=81,17.1 \%)$ were diagnosed at the age less than 30 years, and $(n=32,30.5 \%)$ were diagnosed at the age more than 40 years. Among the presented sociodemographic features presented in Table 1, the difference between the two groups reached a statistical significant level $(\mathrm{p}<0.05)$ only in three factors, namely: number of family members, number of children, and kind of family house, indicating that they affect the chance of getting breast cancer disease and classified as one of the risk factors that affect breast cancer disease among women in the Gaza strip.

\subsection{Risk Factors Associated with Breast Cancer in Univariate Analysis}

The binary logistic regression analysis was applied to define the association between the potential risk factors and incidence of $\mathrm{BC}$ among women in the Gaza strip. The strength of association was achieved using the crude odds ratio (OR) at $95 \%$ CI confidence interval. As presented in Table 2, a number of risk factors related to the history of exposure to toxic gases, radiation, shells and pesticides, anthropometric measurements, and history of medical conditions found to be significantly associated with the incidence of BC $(\mathrm{p}<0.05)$ without controlling for confounders. Regarding exposure to medical radiation, $74.3 \%$ from the 105 cases indicated that they exposed to medical radiation previously compared to $58.1 \%$ from the 210 controls. The difference between the two groups reached a statistically significant level $(\mathrm{COR}=2.2 ; 95 \% \mathrm{CI}: 1.3-3.7)$, which means that 
this factor has the ability to develop breast cancer among women two times more likely. Likewise, the increase of body mass index (BMI) more than 30 $\mathrm{kg} / \mathrm{m}^{2}$ was significantly associated with getting breast cancer $(\mathrm{p}<0.05)$ and 3.6 times more probable to cause breast cancer (COR = 3.6; 95\% CI: 1.8 - 7.5). Additionally, hypertension showed a positive association with the developing of BC $(\mathrm{p}<0.05)$. The chance of getting breast cancer among women who had high blood pressure was three times more than women who did not have hypertension $(\mathrm{COR}=3.0 ; 95 \% \mathrm{CI}: 1.5-6.0)$. Diabetes was found to be positively associated with $\mathrm{BC}$ and it was a risk factor for developing $\mathrm{BC}(\mathrm{COR}=2.3$; 95\% CI: 1.3 - 4.4). However, no significant association was found for the other factors $(\mathrm{p}>0.05)$.

The results in Table 3, present that women age at first pregnancy was significantly associated with $\mathrm{BC}(\mathrm{p}<0.05)$ and a difference between the two groups was shown since the age between 19 and 24 years old was protective factor (COR $=0.5$; 95\% CI: $0.3-0.9$ ) while the age of more than 24 years old was risk factor for developing $\mathrm{BC}(\mathrm{COR}=1.3$; 95\% CI: 0.6 - 3). Also, last pregnancy time showed a statistical significance $(\mathrm{p}<0.05)$. Furthermore, prolonged breastfeeding between 13 and 24 months displayed a statistical significance $(\mathrm{p}<0.05)$ and protecting factor $(\mathrm{COR}=0.3 ; 95 \% \mathrm{CI}$ : $0.2-0.6)$. Likewise, reaching menopause was significantly associated with $\mathrm{BC}(\mathrm{p}<0.05)$ and 3.6 times more likely to develop BC among women in the Gaza strip (COR = 3.6; 95\% CI: 2.2 - 5.9). Nevertheless, the other factors showed no significant association with BC.

Table 4, indicated that a statistically significant relationship between the history of $\mathrm{BC}$ in the family and getting $\mathrm{BC}(\mathrm{p}<0.05)$ with 2.7 times more likely to cause $\mathrm{BC}(\mathrm{COR}=2.7$; 95\% CI: 1.7 - 4.4). As well, getting BC was 3.8 times more probable in the case of a history of incidence of $\mathrm{BC}$ in one of the first-degree relations $(\mathrm{COR}=3.8 ; 95 \% \mathrm{CI}: 1.4-10.3)$. The daily walking exercise was a protective factor of $\mathrm{BC}(\mathrm{COR}=0.6 ; 95 \% \mathrm{CI}: 0.3-1.2)$ and $(\mathrm{COR}=0.4 ; 95 \% \mathrm{CI}: 0.2$ 0.7 ) for daily waking minutes less than 20 and between 20 and 40 minutes, respectively.

As revealed in Table 5, among the included 19 food groups, only sex factors were significantly associated with the incidence of BC among women in the Gaza strip ( $\mathrm{p}<0.05$ ), namely: vegetable oils, snacks, fruit, beans and legumes, sugar, sweets and desserts, and vegetables. Moreover, the number of daily meals also was significantly associated with BC.

\subsection{Risk Factors Associated with Breast Cancer in Multiple Logistic Regression Analysis}

Variables with $\mathrm{p}<0.05$ in the univariate model were inserted into the multivariate model. Many variables missed their association with $\mathrm{BC}$ in the multivariate model under adjustment for all confounders. Table 6, shows that woman with BMI more than or equal $30 \mathrm{~kg} / \mathrm{m}^{2}$ are under risk of getting BC 2.9 times greater than those have BMI less than $29 \mathrm{~kg} / \mathrm{m}^{2}$ (AOR = 2.895; 95\% CI: $1.305-6.423$ ). 
Analysis of risk according to reaching menopause showed that the estimated AOR was greater among those reached menopause (3.137, 95\% CI 1.824 - 5.395) than among those did not reach menopause. The risk of developing $\mathrm{BC}$ in the case of a history of $\mathrm{BC}$ incidence in the family was more than two times higher $(\mathrm{AOR}=2.632,95 \% \mathrm{CI} 1.528-4.535)$ than in case of the history of a free family of BC.

Table 1. Sociodemographic features of the study population.

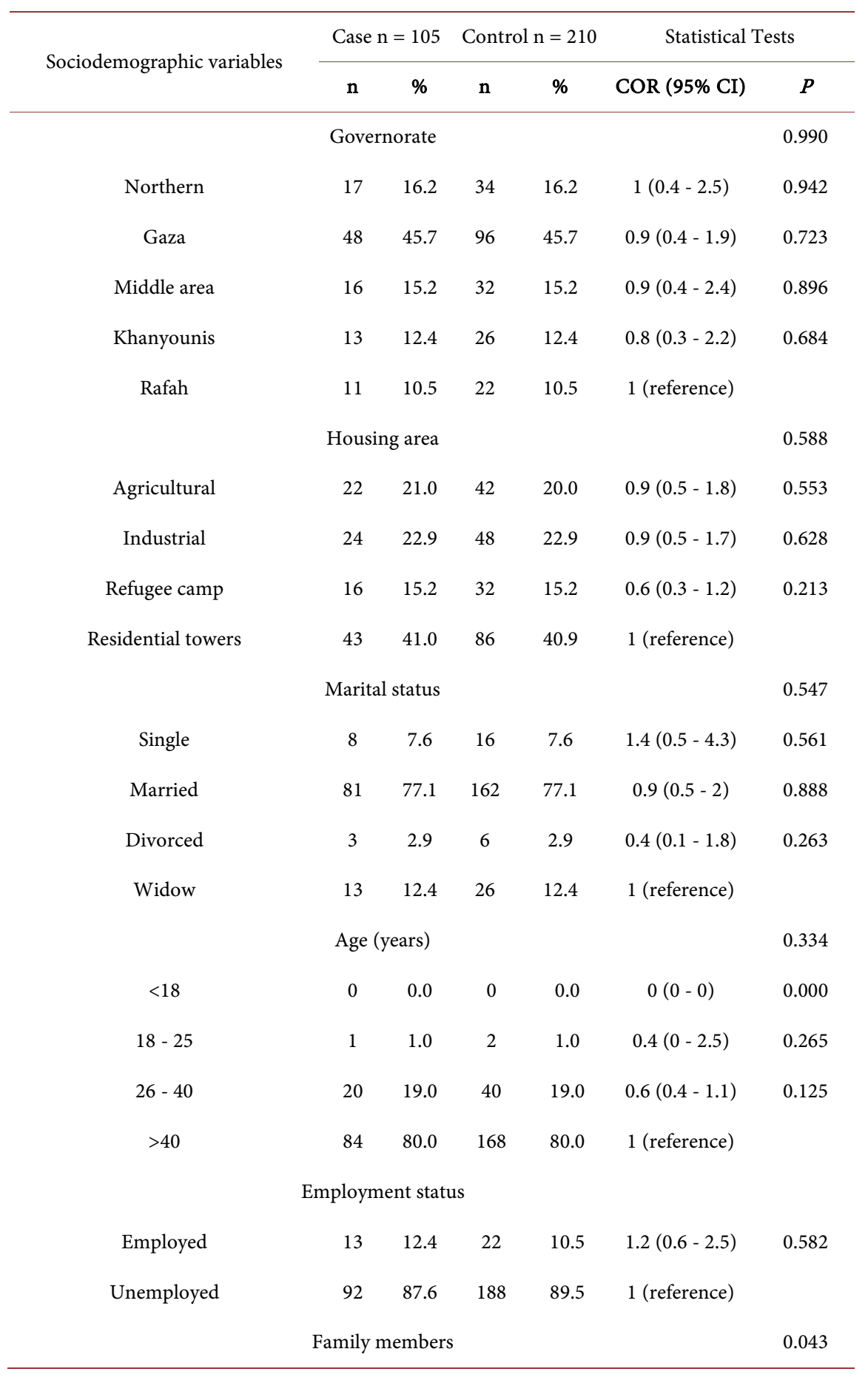




\section{Continued}

\begin{tabular}{|c|c|c|c|c|c|c|}
\hline$<3$ & 28 & 26.7 & 31 & 14.8 & 1 (reference) & \\
\hline $3-5$ & 18 & 17.1 & 46 & 21.9 & $0.4(0.2-0.9)$ & 0.017 \\
\hline $6-8$ & 23 & 21.9 & 63 & 30.0 & $0.4(0.2-0.8)$ & 0.011 \\
\hline$>5$ & 36 & 34.3 & 70 & 33.3 & $0.6(0.3-1.1)$ & 0.090 \\
\hline \multicolumn{6}{|c|}{ Number of children } & 0.011 \\
\hline 0 & 6 & 5.7 & 39 & 18.6 & 1 (reference) & \\
\hline $1-3$ & 24 & 22.9 & 53 & 25.2 & $2.8(1-7.4)$ & 0.043 \\
\hline $4-5$ & 56 & 53.3 & 95 & 45.2 & $3.8(1.5-9.6)$ & 0.004 \\
\hline$>6$ & 19 & 18.1 & 23 & 11.0 & $5.4(1.9-15.4)$ & 0.002 \\
\hline \multicolumn{6}{|c|}{ Educational level } & 0.349 \\
\hline Primary & 19 & 18.1 & 23 & 11.0 & $1.7(0.8-3.8)$ & 0.351 \\
\hline Secondary & 25 & 23.8 & 58 & 27.6 & $0.9(0.5-1.8)$ & 0.680 \\
\hline Diploma & 39 & 37.1 & 83 & 39.5 & $0.9(0.5-1.8)$ & 0.305 \\
\hline University & 22 & 21.0 & 46 & 21.9 & 1 (reference) & \\
\hline \multicolumn{6}{|c|}{ Monthly income (NIS) } & 0.179 \\
\hline$<500$ & 4 & 3.8 & 9 & 4.3 & 1 (reference) & \\
\hline $600-1500$ & 65 & 61.9 & 104 & 49.5 & $1.4(0.4-4.6)$ & 0.611 \\
\hline $1600-2500$ & 22 & 21.0 & 73 & 34.8 & $0.7(0.2-2.4)$ & 0.549 \\
\hline $2600-3500$ & 11 & 10.5 & 20 & 9.5 & $1.2(0.3-5)$ & 0.764 \\
\hline$>3600$ & 3 & 2.9 & 4 & 1.9 & $1.7(0.3-11.3)$ & 0.590 \\
\hline \multicolumn{6}{|c|}{ House type } & 0.036 \\
\hline Villa & 1 & 1.0 & 2 & 1.0 & $3(0.2-37.7)$ & 0.395 \\
\hline Apartment & 49 & 46.6 & 78 & 37.1 & $3.6(1.5-8.8)$ & 0.004 \\
\hline Independent house & 48 & 45.7 & 88 & 41.9 & $3.3(1.4-7.8)$ & 0.008 \\
\hline Asbestos & 7 & 6.7 & 42 & 20.0 & 1 (reference) & \\
\hline \multicolumn{7}{|c|}{ House ownership } \\
\hline Owned & 95 & 90.5 & 178 & 84.8 & $1.7(0.8-3.6)$ & 0.176 \\
\hline Rented & 10 & 9.5 & 32 & 15.2 & 1 (reference) & \\
\hline \multicolumn{7}{|c|}{ Age at breast cancer diagnosis (year) } \\
\hline$<30$ & 18 & 17.1 & & & & \\
\hline $30-40$ & 55 & 52.4 & & & & \\
\hline$>41$ & 32 & 30.5 & & & & \\
\hline
\end{tabular}

$\mathrm{n}=$ Frequency; Ref = Reference category; $\mathrm{COR}=$ Denotes crude odds ratio using $95 \%$ confidence interval in univariate logistic regression analysis; $\mathrm{CI}=$ Confidence interval; $\mathrm{p}<0.05=$ significant on univariate analysis; NIS $=$ Denotes New Israeli Shekel, the local currency ( 1 USD $\approx 3.63$ NIS). 
Table 2. History of exposure to toxic gases, radiation, shells and pesticides, anthropometric measurements, and medical conditions risk factors and BC among women in Gaza strip.

\begin{tabular}{llllllll}
\hline \multirow{2}{*}{ Risk factors } & \multicolumn{2}{c}{ Case $\mathrm{n}=105$} & \multicolumn{2}{c}{ Control $\mathrm{n}=210$} & \multicolumn{2}{c}{ Statistical Tests } \\
\cline { 2 - 7 } & n & $\%$ & n & $\%$ & COR $(95 \% \mathrm{CI})$ & $\boldsymbol{P}$ \\
\hline
\end{tabular}

Previous exposure to toxic gases, radiation, shells, and pesticides

Exposed to toxic gas inhalation

$\begin{array}{lllllll}\text { Yes } & 32 & 30.5 & 70 & 33.3 & 0.9(0.5-1.5) & 0.668 \\ \text { No } & 73 & 69.5 & 140 & 66.7 & 1 \text { (reference) } & \end{array}$

Exposed to medical radiation

$\begin{array}{lllllll}\text { Yes } & 78 & 74.3 & 122 & 58.1 & 2.2(1.3-3.7) & 0.003 \\ \text { No } & 27 & 25.7 & 88 & 41.9 & 1 \text { (reference) } & \end{array}$

Your house or surrounding area exposed to shells

$\begin{array}{llllll}\text { Yes } & 36 & 34.3 & 77 & 36.7 & 0.9(0.6-1.5) \\ \text { No } & 69 & 65.7 & 133 & 63.3 & 1 \text { (reference) }\end{array}$

Exposed to pesticides

$\begin{array}{lllllll}\text { Yes } & 18 & 17.1 & 42 & 20.0 & 0.8(0.5-1.6) & 0.581 \\ \text { No } & 87 & 82.9 & 168 & 80.0 & 1 \text { (reference) } & \end{array}$

Anthropometric measurements

Body mass index (BMI)

$$
\begin{aligned}
& \geq 30 \mathrm{~kg} / \mathrm{m}^{2} \\
& <29 \mathrm{~kg} / \mathrm{m}^{2}
\end{aligned}
$$

Yes

No

Yes

No

Yes

No

Yes

No

Inflammatory diseases

$\begin{array}{llllll}\text { Yes } & 17 & 16.2 & 41 & 20.6 & 0.8(0.4-1.4) \\ \text { No } & 88 & 83.8 & 158 & 79.4 & 1 \text { (reference) }\end{array}$

$\begin{array}{lllll}10 & 9.5 & 59 & 28.1 & 1 \text { (reference) }\end{array}$

History of medical conditions

High blood pressure

\begin{tabular}{lrrrrr}
22 & 21.0 & 16 & 8.2 & $3.0(1.5-6.0)$ & 0.002 \\
83 & 79.0 & 178 & 91.8 & 1 (reference) & \\
\multicolumn{2}{l}{ Diabetes } & & & & \\
& 26 & 24.8 & 13 & $2.3(1.3-4.4)$ & 0.001 \\
& & & & &
\end{tabular}

Cardiac diseases

\begin{tabular}{lccccc}
11 & 10.5 & 17 & 8.1 & $1.3(0.6-3)$ & 0.461 \\
94 & 89.5 & 193 & 91.9 & 1 (reference) & \\
\multicolumn{2}{c}{ Anemia } & & & & \\
6 & 5.7 & 20 & 10.1 & $0.5(0.2-1.4)$ & 0.212 \\
99 & 94.3 & 178 & 89.9 & 1 (reference)
\end{tabular}

0.001

0.383 
Table 3. Maternal and reproductive health risk factors and BC among women in Gaza strip.

\begin{tabular}{|c|c|c|c|c|c|c|}
\hline \multirow{2}{*}{ Risk factors } & \multicolumn{2}{|c|}{ Case $\mathrm{n}=105$} & \multicolumn{2}{|c|}{ Control $n=210$} & \multicolumn{2}{|c|}{ Statistical Tests } \\
\hline & $\mathbf{n}$ & $\%$ & $\mathbf{n}$ & $\%$ & $\operatorname{COR}(95 \% \mathrm{CI})$ & $P$ \\
\hline \multicolumn{7}{|c|}{ 1) Maternal and reproductive health } \\
\hline \multicolumn{7}{|c|}{ Marriage age (year) } \\
\hline$\leq 18$ & 52 & 49.5 & 91 & 43.3 & $1.2(0.8-2)$ & 0.362 \\
\hline$>19$ & 53 & 50.5 & 119 & 56.7 & 1 (reference) & \\
\hline \multicolumn{6}{|c|}{ Age at the first menstruation (year) } & 0.150 \\
\hline$<12$ & 11 & 10.5 & 1 & 5.7 & $0.5(0.2-1.3)$ & 0.163 \\
\hline $13-16$ & 84 & 80.0 & 166 & 79.0 & $0.3(0.1-1)$ & 0.052 \\
\hline$<16$ & 10 & 9.5 & 32 & 15.3 & 1 (reference) & \\
\hline Age at the first pregnar & & & & & & 0.017 \\
\hline$<18$ & 25 & 29.4 & 39 & 20.6 & 1 (reference) & \\
\hline $19-24$ & 44 & 51.8 & 133 & 70.4 & $0.5(0.3-0.9)$ & 0.033 \\
\hline$>24$ & 16 & 18.8 & 17 & 9.0 & $1.3(0.6-3)$ & 0.545 \\
\hline \multicolumn{7}{|c|}{ Number of pregnancies } \\
\hline$\leq 4$ & 69 & 65.7 & 126 & 60.0 & $1.2(0.8-2)$ & 0.372 \\
\hline$>5$ & 36 & 34.3 & 84 & 40.0 & 1 (reference) & \\
\hline \multicolumn{7}{|c|}{ Number of deliveries } \\
\hline$\leq 4$ & 68 & 64.8 & 121 & 57.6 & $1.3(0.8-2.1)$ & 0.261 \\
\hline$>5$ & 37 & 35.2 & 89 & 42.4 & 1 (reference) & \\
\hline \multicolumn{7}{|c|}{ Number of abortions } \\
\hline$\leq 2$ & 78 & 74.3 & 170 & 81.0 & 1 (reference) & 0.257 \\
\hline$>3$ & 27 & 25.7 & 40 & 19.0 & $1.4(0.8-2.4)$ & \\
\hline \multicolumn{7}{|c|}{ The period between pregnancies (year) } \\
\hline$\leq 1$ & 19 & 23.5 & 50 & 31.8 & 1 (reference) & 0.206 \\
\hline$>2$ & 62 & 76.5 & 107 & 68.2 & $1.5(0.8-2.7)$ & \\
\hline \multicolumn{6}{|c|}{ Last pregnancy time (month) } & 0.002 \\
\hline$<18$ & 41 & 42.7 & 72 & 36.2 & 1 (reference) & \\
\hline $19-24$ & 36 & 37.5 & 113 & 56.8 & $0.6(0.3-0.99)$ & 0.034 \\
\hline$>24$ & 19 & 19.8 & 14 & 7.0 & $2.1(1-4.6)$ & 0.063 \\
\hline \multicolumn{6}{|c|}{ Use natural breastfeeding } & 0.198 \\
\hline Yes & 77 & 87.5 & 177 & 92.2 & $0.6(0.3-1.3)$ & \\
\hline No & 11 & 12.5 & 15 & 7.8 & 1 (reference) & \\
\hline \multicolumn{7}{|c|}{ Difficulties in breastfeeding } \\
\hline Yes & 15 & 17.0 & 25 & 13.0 & $1.2(0.6-2.5)$ & 0.562 \\
\hline No & 73 & 83.0 & 167 & 87.0 & 1 (reference) & \\
\hline
\end{tabular}




\section{Continued}

\begin{tabular}{|c|c|c|c|c|c|c|}
\hline \multicolumn{6}{|c|}{ Weaning time (month) } & \multirow[t]{2}{*}{0.002} \\
\hline$\leq 6$ & 7 & 8.0 & 15 & 7.8 & 1 (reference) & \\
\hline $7-12$ & 16 & 18.1 & 77 & 40.1 & $0.7(0.3-1.9)$ & 0.528 \\
\hline $13-24$ & 65 & 73.9 & 100 & 52.1 & $0.3(0.2-0.6)$ & 0.001 \\
\hline \multicolumn{7}{|c|}{ Reached menopause } \\
\hline Yes & 63 & 60.0 & 60 & 28.6 & $3.6(2.2-5.9)$ & 0.001 \\
\hline No & 42 & 40.0 & 150 & 71.4 & 1 (reference) & \\
\hline \multicolumn{7}{|c|}{ Age at menopause (year) } \\
\hline$\leq 45$ & 31 & 40.3 & 30 & 42.3 & 1 (reference) & 0.658 \\
\hline$>46$ & 46 & 59.7 & 41 & 57.7 & $1.2(0.6-2.2)$ & \\
\hline \multicolumn{7}{|c|}{ Use of contraceptives } \\
\hline Yes & 27 & 28.1 & 57 & 28.8 & $0.9(0.5-1.6)$ & 0.752 \\
\hline No & 69 & 71.9 & 141 & 71.2 & 1 (reference) & \\
\hline \multicolumn{7}{|c|}{ Preeclampsia } \\
\hline Yes & 4 & 3.8 & 17 & 8.6 & $0.3(0.1-1.0)$ & 0.056 \\
\hline No & 101 & 96.2 & 180 & 91.4 & 1 (reference) & \\
\hline \multicolumn{7}{|c|}{ Supplements during pregnancy } \\
\hline Yes & 49 & 46.7 & 119 & 56.7 & $0.7(0.4-1.1)$ & 0.121 \\
\hline No & 56 & 53.3 & 9 & 43.3 & 1 (reference) & \\
\hline \multicolumn{6}{|c|}{ If yes, what kind } & 0.021 \\
\hline Iron & 17 & 37.8 & 67 & 62.0 & $4.7(1.8-12.8)$ & 0.002 \\
\hline Prenatal Vitamins & 12 & 26.7 & 10 & 9.3 & $0.9(0.2-4.4)$ & 0.873 \\
\hline Folate & 2 & 4.4 & 9 & 8.3 & $2.4(0.9-6.3)$ & 0.086 \\
\hline Fish Oil & 9 & 20.0 & 15 & 13.9 & $2.8(0.8-10)$ & 0.109 \\
\hline Others & 5 & 11.1 & 7 & 6.5 & 1 (reference) & \\
\hline
\end{tabular}

Table 4. Family history of cancer and life style risk factors and BC among women in Gaza strip.

\begin{tabular}{llllllll}
\hline \multirow{2}{*}{ Risk factors } & \multicolumn{2}{c}{ Case $\mathrm{n}=105$} & Control $\mathrm{n}=210$ & \multicolumn{2}{c}{ Statistical Tests } \\
\cline { 2 - 7 } & $\mathbf{n}$ & $\%$ & $\mathbf{n}$ & $\%$ & COR (95\% CI) & $\boldsymbol{P}$ \\
\hline
\end{tabular}

1) Family history of cancer

One member of the family had cancer

$\begin{array}{lrrrrrr}\text { Yes } & 38 & 36.2 & 63 & 30.0 & 1.3(0.8-2.1 & 0.349 \\ \text { No } & 67 & 63.8 & 147 & 70.0 & 1 \text { (reference) } & \end{array}$

If yes, degree of relation

$\begin{array}{ccccccc}\text { First degree } & 48 & 82.8 & 53 & 70.7 & 1.9(0.8-4.5) & 0.131 \\ \text { Second degree } & 10 & 17.2 & 22 & 29.3 & 1 \text { (reference) } & \end{array}$




\section{Continued}

\begin{tabular}{|c|c|c|c|c|c|c|}
\hline \multicolumn{7}{|c|}{ One member of the family had breast cancer } \\
\hline Yes & 58 & 55.2 & 64 & 30.5 & $2.7(1.7-4.4)$ & 0.001 \\
\hline No & 47 & 44.8 & 146 & 69.5 & 1 (reference) & \\
\hline \multicolumn{7}{|c|}{ If yes, degree of relation } \\
\hline First degree & 31 & 81.6 & 42 & 58.3 & $3.8(1.4-10.3)$ & 0.008 \\
\hline Second degree & 7 & 18.4 & 30 & 41.7 & 1 (reference) & \\
\hline \multicolumn{7}{|c|}{ 2) Life style } \\
\hline \multicolumn{7}{|c|}{ Smoker } \\
\hline Yes & 2 & 1.9 & 7 & 3.3 & $1.7(0.4-8.6)$ & 0.490 \\
\hline No & 103 & 98.1 & 203 & 96.7 & 1 (reference) & \\
\hline \multicolumn{7}{|c|}{ Expose to secondhand smoke } \\
\hline Yes & 54 & 51.4 & 86 & 41.0 & $1.6(1-2.5)$ & 0.061 \\
\hline No & 51 & 48.6 & 124 & 59.0 & 1 (reference) & \\
\hline \multicolumn{6}{|c|}{ Daily walking (minute) } & 0.013 \\
\hline$<20$ & 16 & 20.3 & 33 & 23.2 & $0.6(0.3-1.2)$ & 0.117 \\
\hline $20-40$ & 19 & 24.1 & 58 & 40.8 & $0.4(0.2-0.7)$ & 0.004 \\
\hline$>41$ & 44 & 55.7 & 51 & 35.9 & 1 (reference) & \\
\hline \multicolumn{6}{|c|}{ Sleeping hours } & 0.156 \\
\hline$<6$ & 14 & 13.3 & 12 & 7.8 & $2.4(1.0-6.2)$ & 0.060 \\
\hline $6-8$ & 70 & 66.7 & 97 & 63.4 & $1.5(0.8-2.7)$ & 0.207 \\
\hline$>8$ & 21 & 20.0 & 44 & 28.8 & 1 (reference) & \\
\hline \multicolumn{7}{|c|}{ Monthly breast self-exam } \\
\hline Yes & 17 & 16.2 & 24 & 11.4 & $1.5(0.8-3)$ & 0.220 \\
\hline No & 88 & 83.8 & 186 & 88.6 & 1 (reference) & \\
\hline
\end{tabular}

Table 5. Univariate model of dietary habits and their association with breast cancer among women in Gaza strip.

\begin{tabular}{|c|c|c|c|c|c|c|}
\hline \multirow{2}{*}{ Food groups } & \multicolumn{2}{|c|}{ Case $\mathrm{n}=105$} & \multicolumn{2}{|c|}{ Control $\mathrm{n}=210$} & \multicolumn{2}{|c|}{ Statistical Tests } \\
\hline & $\mathbf{n}$ & $\%$ & $\mathbf{n}$ & $\%$ & $\operatorname{COR}(95 \% \mathrm{CI})$ & $P$ \\
\hline \multicolumn{6}{|c|}{ High - fat dairy products } & 0.449 \\
\hline Daily & 34 & 32.4 & 67 & 31.9 & $0.7(0.3-1.3)$ & 0.262 \\
\hline 2 - 3 times weekly & 32 & 30.5 & 68 & 32.4 & $0.6(0.3-1.3)$ & 0.228 \\
\hline Weekly & 13 & 12.4 & 26 & 12.4 & $0.7(0.3-1.6)$ & 0.405 \\
\hline Monthly & 5 & 4.8 & 20 & 9.5 & $0.3(0.1-1.1)$ & 0.065 \\
\hline Never & 21 & 20.0 & 29 & 13.8 & 1 (reference) & \\
\hline \multicolumn{6}{|c|}{ Eggs } & 0.138 \\
\hline Daily & 20 & 19.2 & 53 & 25.4 & $1.4(0.3-5.5)$ & 0.644 \\
\hline 2 - 3 times weekly & 61 & 58.7 & 91 & 43.5 & $2.4(0.6-8.9)$ & 0.195 \\
\hline Weekly & 13 & 12.5 & 42 & 20.1 & $1.1(0.3-4.7)$ & 0.861 \\
\hline Monthly & 7 & 6.7 & 12 & 5.7 & $2.1(0.4-10.4)$ & 0.346 \\
\hline Never & 3 & 2.9 & 11 & 5.3 & 1 (reference) & \\
\hline
\end{tabular}




\section{Continued}

\begin{tabular}{|c|c|c|c|c|c|c|}
\hline \multicolumn{6}{|c|}{ Red meat } & \multirow{2}{*}{$\begin{array}{l}0.108 \\
0.503\end{array}$} \\
\hline Daily & 11 & 10.5 & 10 & 4.8 & $1.4(0.5-4.2)$ & \\
\hline 2 - 3 times weekly & 31 & 29.5 & 59 & 28.1 & $0.7(0.3-1.5)$ & 0.352 \\
\hline Weekly & 32 & 30.5 & 76 & 36.1 & $0.5(0.2-1.1)$ & 0.106 \\
\hline Monthly & 15 & 14.3 & 44 & 21.0 & $0.4(0.2-1.1)$ & 0.072 \\
\hline \multirow[t]{2}{*}{ Never } & 16 & 15.2 & 21 & 10.0 & 1 (reference) & 0.503 \\
\hline & \multicolumn{3}{|c|}{ Organ meat } & & & 0.157 \\
\hline Daily & 21 & 20.0 & 53 & 25.4 & $1.5(0.4-5.7)$ & 0.594 \\
\hline 2 - 3 times weekly & 61 & 58.1 & 91 & 43.5 & $2.4(0.6-8.9)$ & 0.195 \\
\hline Weekly & 13 & 12.3 & 42 & 20.1 & $1.1(0.3-4.7)$ & 0.861 \\
\hline Monthly & 7 & 6.7 & 12 & 5.7 & $2.1(0.4-10.4)$ & 0.346 \\
\hline \multirow[t]{2}{*}{ Never } & 3 & 2.9 & 11 & 5.3 & 1 (reference) & \\
\hline & \multicolumn{4}{|c|}{ High-fat dairy products } & & 0.108 \\
\hline Daily & 11 & 10.5 & 10 & 4.8 & $1.4(0.5-4.2)$ & 0.503 \\
\hline 2 - 3 times weekly & 31 & 29.5 & 59 & 28.1 & $0.7(0.3-1.5)$ & 0.352 \\
\hline Weekly & 32 & 30.5 & 76 & 36.2 & $0.5(0.2-1.1)$ & 0.106 \\
\hline Monthly & 15 & 14.3 & 44 & 21.0 & $0.4(0.2-1.1)$ & 0.072 \\
\hline \multirow[t]{2}{*}{ Never } & 16 & 15.2 & 21 & 10.0 & 1 (reference) & \\
\hline & \multicolumn{3}{|c|}{ Hydrogenated fats } & & & 0.171 \\
\hline Daily & 4 & 3.8 & 17 & 8.1 & $0.3(0.1-1.1)$ & 0.071 \\
\hline 2 - 3 times weekly & 12 & 11.4 & 30 & 14.3 & $0.6(0.3-1.3)$ & 0.173 \\
\hline Weekly & 21 & 20.0 & 45 & 21.4 & $0.6(0.3-1.2)$ & 0.170 \\
\hline Monthly & 27 & 13.2 & 38 & 18.1 & $0.5(0.3-1.1)$ & 0.092 \\
\hline \multirow[t]{2}{*}{ Never } & 54 & 51.4 & 80 & 38.1 & 1 (reference) & 0.071 \\
\hline & \multicolumn{3}{|c|}{ Vegetable oils } & & & 0.025 \\
\hline Daily & 7 & 6.7 & 21 & 10.0 & $0.4(0.1-0.9)$ & 0.036 \\
\hline 2 - 3 times weekly & 9 & 8.6 & 19 & 9.0 & $0.6(0.3-1.5)$ & 0.272 \\
\hline Weekly & 11 & 10.5 & 42 & 20.0 & $0.3(0.2-0.7)$ & 0.005 \\
\hline Monthly & 27 & 25.7 & 62 & 29.5 & $0.6(0.3-1)$ & 0.053 \\
\hline \multirow[t]{2}{*}{ Never } & 51 & 48.5 & 66 & 31.5 & 1 (reference) & \\
\hline & \multicolumn{3}{|c|}{ Poultry } & & & 0.125 \\
\hline Daily & 4 & 3.8 & 13 & 6.2 & $2.3(0.4-14.7)$ & 0.376 \\
\hline 2 - 3 times weekly & 27 & 25.7 & 42 & 20.0 & $4.8(1-22.8)$ & 0.047 \\
\hline Weekly & 61 & 58.1 & 105 & 50.0 & $4.2(0.9-19.2)$ & 0.060 \\
\hline Monthly & 11 & 10.5 & 35 & 16.7 & $2.4(0.5-12)$ & 0.301 \\
\hline \multirow[t]{2}{*}{ Never } & 2 & 1.9 & 15 & 7.1 & 1 (reference) & \\
\hline & \multicolumn{3}{|c|}{ Beverages } & & & 0.672 \\
\hline Daily & 7 & 6.7 & 13 & 6.2 & $1.1(0.4-3)$ & 0.924 \\
\hline 2 - 3 times weekly & 7 & 6.7 & 17 & 8.1 & $1(0.4-2.7)$ & 0.983 \\
\hline Weekly & 21 & 20.0 & 39 & 18.6 & $1.3(0.7-2.6)$ & 0.413 \\
\hline Monthly & 37 & 35.2 & 60 & 28.6 & $1.5(0.9-2.7)$ & 0.158 \\
\hline Never & 33 & 31.4 & 81 & 38.5 & 1 (reference) & \\
\hline
\end{tabular}




\section{Continued}

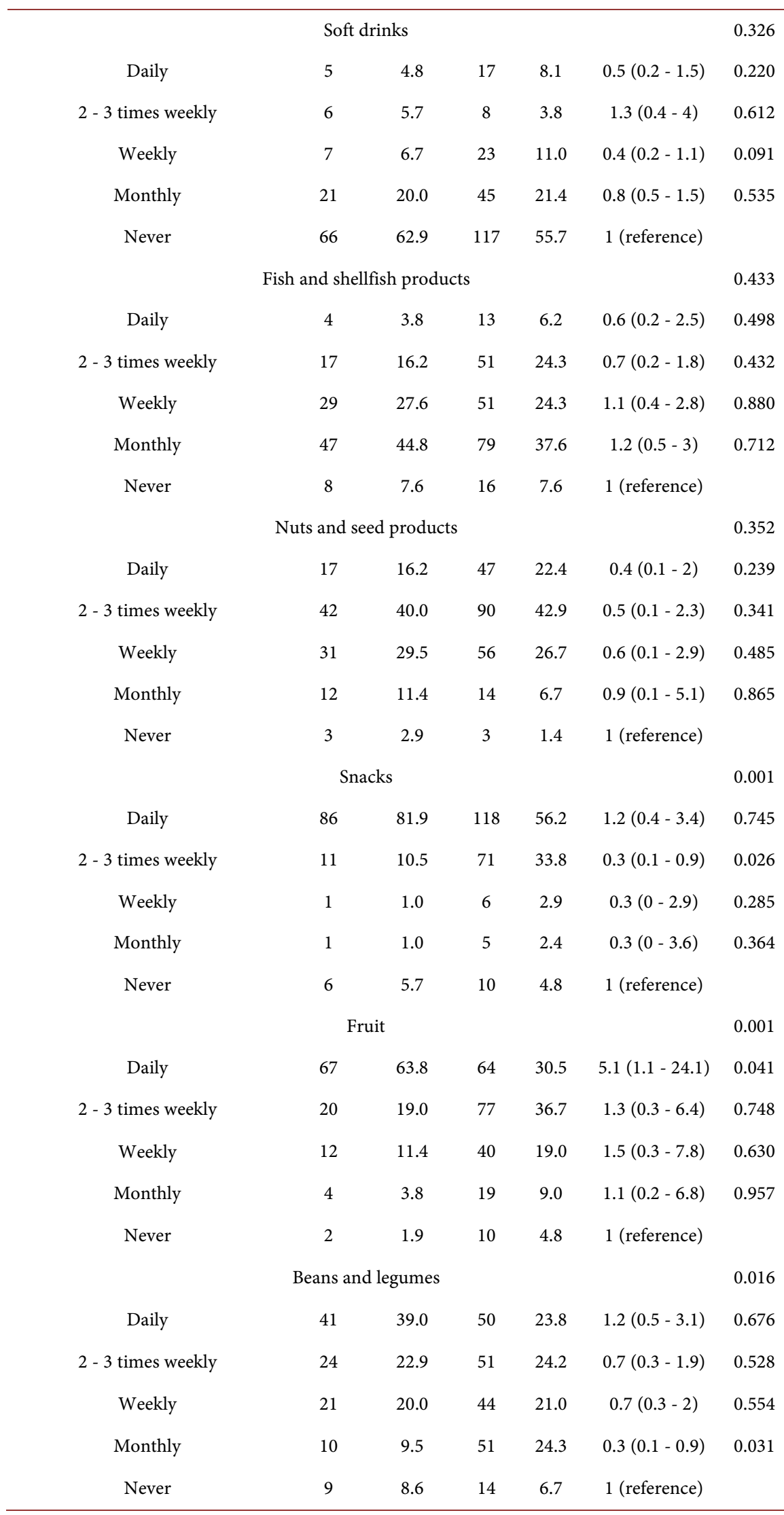




\section{Continued}

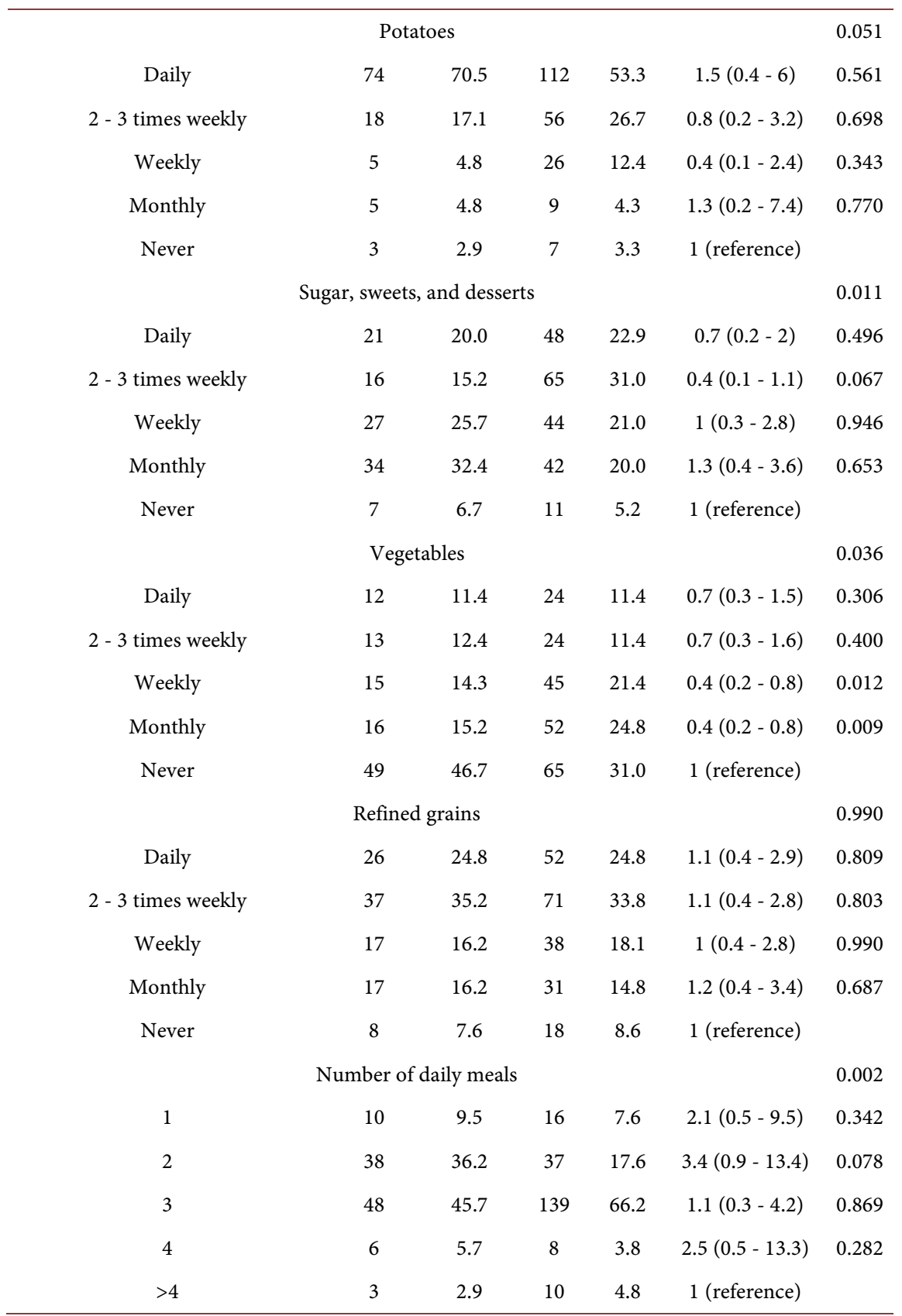

Table 6. Multiple logistic regression model of risk factors and BC among women in Gaza strip.

\begin{tabular}{cccccc}
\hline Factors & B & S.E. & Wald & $P$ & AOR (95\% CI) \\
\hline Body mass index & 1.1 & 0.4 & 6.8 & 0.009 & $2.895(1.305-6.423)$ \\
Reached menopause & 1.1 & 0.3 & 17.1 & $<0.001$ & $3.137(1.824-5.395)$ \\
One member of the family had breast cancer & 1.0 & 0.3 & 12.2 & $<0.001$ & $2.632(1.528-4.535)$ \\
Constant & $2.4-$ & 0.5 & 28.0 & $<0.001$ & 0.087 \\
\hline
\end{tabular}

AOR = Denotes adjusted odds ratio using 95\% confidence interval in multivariable logistic regression analysis; $\mathrm{B}=$ slope $\mathrm{CI}=$ Confidence interval; $\mathrm{p}<0.05$ : Significant, $\mathrm{p}>0.05$ : Not significant. 


\section{Discussion}

The primary purpose of knowing the risk factors related to breast cancer is to take precautionary measures to prevent the incidence of breast cancer. Therefore, we investigate potential risk factors for breast cancer among women in the Gaza strip, Palestine. The outcomes of this study, in the multivariate model, have indicated three risk factors associated with the incidence of BC among Gazan's women namely: high BMI more than or equal $30 \mathrm{~kg} / \mathrm{m}^{2}$, reaching menopause, and history of $\mathrm{BC}$ incidence in the family. On the other hand, in the univariate model, several factors seemed to be risk factors for BC.

Previous epidemiological studies have reported that premenopausal obesity is generally protective for breast cancer [20], while postmenopausal obesity is associated with increased risk [21]. Our case-control analysis revealed that patients with a BMI of $30 \mathrm{~kg} / \mathrm{m}^{2}$ or more had more chance for getting disease compared with patients with a BMI below $29 \mathrm{~kg} / \mathrm{m}^{2}$ ( $\left.\mathrm{p}<0.001\right)$. Our findings in this regard are in line with the results of former research in other countries [22] [23] [24]. Moreover, there is no evidence that the assumption of a simple linear or a log-linear relationship between BMI and BC risk is real, in particular when BMI is less than $25 \mathrm{~kg} / \mathrm{m}^{2}$ [25]. The mechanisms underlying the relationship between high BMI and BC in women was discussed in the literature and it was primarily the result of the associated increase in estrogens, particularly bioavailable estradiol [22] [26].

With respect to menopause age and risk of breast cancer in women, findings of the present study also indicated that reaching menopause was significantly associated with developing of breast cancer among women in Gaza strip. Similar outcomes were reported in previous studies stated that breast cancer is a disease of older women and its incidence increases with age, and it is rare below the age of 20 years [27] [28] [29] [30]. More than half of patients in our study were between the third and fourth decade of their life, in contrast to the western countries where only $23 \%$ of women younger than 40 years presented with breast cancer [31].

The significant association between the family history and BC among women in Gaza strip indicated to imply a genetic predisposition [32]. We herein, recommend more investigation to find out what gene linked to BC. In any case, the literature indicates a family history of $\mathrm{BC}$ in any first-degree relative is known to increase a women risk of disease onset [32] [33] [34] [35]. In addition, these results are consistent with the work of Buxton et al., [36], Caruso et al., [37], Silk et al., [38], Aljohani et al., [39], and a meta-analytic review by Katapodi et al., [40] all of whom found an association between risk perception and family history of breast cancer. In developed countries, about twenty-five percent of inter-individual differences mainly due to genetic causes of BC [41]. Our finding regarding the significant association between the exposure to ionizing radiation and the initiation of breast carcinoma was compatible with the results of previous research [42] [43].

According to the results of previous research, exposure to ionizing radiation 
after 40 years of age does not significantly influence the genesis of breast cancer, but exposure to radiation before 20 years of age significantly influences the initiation of breast carcinoma [27]. An investigation indicated that exposure to diagnostic radiation is accountable for $29 \mathrm{BC}$ cases per year in women in the UK, aged up to 75 years [44].

Our study indicated that there is no association between breast cancer and exposure to pesticides, although there is growing scientific evidence of a link between exposure to pesticides and increased incidence of breast cancer [45].

The present study findings in the univariate model revealed that diabetes and hypertension were a risk factors for $\mathrm{BC}$, this results were consistent with the conclusion of the Boyle et al., meta-analysis found a significantly increased risk of breast cancer among women with diabetes, furthermore, the mechanisms that could increase the breast cancer risk were discussed [46]. Though our study had limited power, our result warrants further investigation and future studies should stratify their analyses by menopausal status particularly because of the increase of chronic diseases epidemics such as hypertension and diabetes among Palestinian population [47]. The potential for an increased risk of BC in women with hypertension has been the subject of a great deal of recent research. A strong correlation between hypertension and BC in women has concluded in some earlier studies [48] [49] [50] [51], however weak relationship was reported in other research [50] [51]. Furthermore, several studies showed that the association was confounded by obesity and high BMI [52] [53].

In this study, the association between BC and intake of different food groups such as vegetable oils, snacks, fruit, beans and legumes, sugar, sweets and desserts, and vegetables. However, other studies revealed that intakes of cereals and grains, vegetables and beans are associated with the reduction in risk of early-stage breast cancer among young women [54]. High risk was shown for all types of meat and fish intake, whereas intakes of eggs and milk were associated with a decreased risk of breast cancer [55].

\section{Conclusion}

Knowing the risk factors for breast cancer may help take preventive measures to reduce the likelihood of developing the disease. Our survey shows that women with body mass index, reaching menopause, and history of $\mathrm{BC}$ in the family are the three main risk factors of BC among women in Gaza strip. Here, educational programs target at women living in Gaza to make them aware and address the misconceptions of the $\mathrm{BC}$ risk factors. Moreover, campaigns to promote the concept of screening for breast cancer among both public and healthcare sectors are critical to improving the rates of early detection of breast cancer in Gaza in order to be able to save lives and for reinforcing societal positive attitudes towards breast health care, including support from family and friends.

\section{Study Limitation}

Some patients did not respond to questionnaire and reject to participate in the study. 


\section{Authors' Contributions}

SY and MY participated in the design of the study and data collection. MD and AM performed the statistical analysis and drafted the manuscript. SA supervised the study and participated in draft review. All authors have read and approved the final version of the manuscript and agree with the order of presentation of the authors.

\section{Acknowledgements}

The authors are grateful to the Palestinian Ministry of Health for giving us a permission to conduct the study in Gaza strip. Also, women who participated in our study are thanked.

\section{Conflicts of Interest}

The authors declare no conflicts of interest regarding the publication of this paper.

\section{References}

[1] WHO. International Agency for Research in Cancer. Globocan. (2012) Estimated Incidence, Mortality and Prevalence Worldwide in 2012, IARC CancerBase No. 11.

[2] Jemal, A., Bray, F., Center, M.M., et al. (2011) Global Cancer Statistics. CA: A Cancer Journal for Clinicians, 61, 69-90. https://doi.org/10.3322/caac.20107

[3] Servick, K. (2014) Breast Cancer: A World of Differences. Science, 343, 1452-1453.

[4] Coughlin, S.S. and Ekwueme, D.U. (2009) Breast Cancer as a Global Health Concern. Cancer Epidemiology, 33, 315-318.

https://doi.org/10.1016/j.canep.2009.10.003

[5] Block, G., Patterson, B. and Subar, A. (1992) Fruit, Vegetables, and Cancer Prevention: A Review of the Epidemiological Evidence. Nutrition and Cancer, 18, 1-29. https://doi.org/10.1080/01635589209514201

[6] Kim, H.J., Jung, S., Eliassen, A.H., et al. (2017) Alcohol Consumption and Breast Cancer Risk in Younger Women according to Family History of Breast Cancer and Folate Intake. American Journal of Epidemiology, 186, 524-531. https://doi.org/10.1093/aje/kwx137

[7] Jemal, A., Center, M.M., De Santis, C., et al. (2010) Global Patterns of Cancer Incidence and Mortality Rates and Trends. Cancer Epidemiology and Prevention Biomarkers, 19, 1893-1907. https://doi.org/10.1158/1055-9965.EPI-10-0437

[8] Elsawaf, Z., Sinn, H.P., Rom, J., et al. (2013) Biological Subtypes of Triple-Negative Breast Cancer Are Associated with Distinct Morphological Changes and Clinical Behaviour. The Breast, 22, 986-992. https://doi.org/10.1016/j.breast.2013.05.012

[9] Phillips, K.A., Steel, E.J., Collins, I., et al. (2016) Transitioning to Routine Breast Cancer Risk Assessment and Management in Primary Care: What Can We Learn from Cardiovascular Disease? Australian Journal of Primary Health, 22, 255-261. https://doi.org/10.1071/PY14156

[10] Aleksoska, K. (2005) Macedonian Women and Breast Cancer: A Model of Psychosocial Support for Early Detection and Treatment. Australian Journal of Primary Health, 11, 63-71. https://doi.org/10.1071/PY05044 
[11] El Saghir, N.S., Khalil, M.K., Eid, T., et al. (2007) Trends in Epidemiology and Management of Breast Cancer in Developing Arab Countries: A Literature and Registry Analysis. International Journal of Surgery, 5, 225-233. https://doi.org/10.1016/j.ijsu.2006.06.015

[12] Barnard, M.E., Boeke, C.E. and Tamimi, R.M. (2015) Established Breast Cancer Risk Factors and Risk of Intrinsic Tumor Subtypes. Biochimica et Biophysica Acta (BBA)-Reviews on Cancer, 1856, 73-85.

[13] De Santis, C., Ma, J., Bryan, L., et al. (2014) Breast Cancer Statistics, 2013. CA: A Cancer Journal for Clinicians, 64, 52-62. https://doi.org/10.3322/caac.21203

[14] Younis, M., Al-Rubaye, D., Haddad, H., et al. (2016) Knowledge and Awareness of Breast Cancer among Young Women in the United Arab Emirates. Advances in Breast Cancer Research, 5, 163. https://doi.org/10.4236/abcr.2016.54019

[15] Farmer, P., Frenk, J., Knaul, F.M., et al. (2010) Expansion of Cancer Care and Control in Countries of Low and Middle Income: A Call to Action. The Lancet, 376, 1186-1193. https://doi.org/10.1016/S0140-6736(10)61152-X

[16] Cuzick, J., Powles, T., Veronesi, U., et al. (2003) Overview of the Main Outcomes in Breast-Cancer Prevention Trials. The Lancet, 36, 296-300. https://doi.org/10.1016/S0140-6736(03)12342-2

[17] Oeffinger, K.C., Fontham, E.T., Etzioni, R., et al. (2015) Breast Cancer Screening for Women at Average Risk: 2015 Guideline Update from the American Cancer Society. JAMA, 314, 1599-1614. https://doi.org/10.1001/jama.2015.12783

[18] MOH Health Status in Palestine (2017) Annual Report 2016. Ministry of Health, Gaza.

[19] Hamdan, M., Monteagudo, C., Lorenzo-Tovar, M.L., et al. (2014) Development and Validation of a Nutritional Questionnaire for the Palestine Population. Public Health Nutrition, 17, 2512-2518. https://doi.org/10.1017/S1368980013002711

[20] Ursin, G., Longnecker, M.P., Haile, R.W., et al. (1995) A Meta-Analysis of Body Mass Index and Risk of Premenopausal Breast Cancer. Epidemiology, 6, 137-141. https://doi.org/10.1097/00001648-199503000-00009

[21] Lahmann, P.H., Hoffmann, K., Allen, N., et al. (2004) Body Size and Breast Cancer Risk: Findings from the European Prospective Investigation into Cancer and Nutrition (EPIC). International Journal of Cancer, 111, 762-771. https://doi.org/10.1002/ijc.20315

[22] Nichols, H.B., Schoemaker, M.J., Wright, L.B., et al. (2017) The Premenopausal Breast Cancer Collaboration: A Pooling Project of Studies Participating in the National Cancer Institute Cohort Consortium. Cancer Epidemiology and Prevention Biomarkers, 26, 1360-1369.

[23] Norsa adah, B., Rusli, B.N., Imran, A.K., et al. (2005) Risk Factors of Breast Cancer in Women in Kelantan. Malaysia Singapore Medical Journal, 46, 698.

[24] Ozmen, V., Ozcinar, B., Karanlik, H., et al. (2009) Breast Cancer Risk Factors in Turkish Women-A University Hospital Based Nested Case Control Study. World Journal of Surgical Oncology, 7, 37. https://doi.org/10.1186/1477-7819-7-37

[25] Bianchini, F., Kaaks, R. and Vainio, H. (2002) Weight Control and Physical Activity in Cancer Prevention. Obesity Reviews, 3, 5-8. https://doi.org/10.1046/j.1467-789X.2002.00046.x

[26] Carpenter, C.L., Ross, R.K., Paganini-Hill, A., et al. (2003) Effect of Family History, Obesity and Exercise on Breast Cancer Risk among Postmenopausal Women. International Journal of Cancer, 106, 96-102. 
[27] Sandhu, D.S., Sandhu, S., Karwasra, R.K., et al. (2010) Profile of Breast Cancer Patients at a Tertiary Care Hospital in North India. Indian Journal of Cancer, 47, 16. https://doi.org/10.4103/0019-509X.58853

[28] Pathy, N.B., Yip, C.H., Taib, N.A., et al. (2011) Breast Cancer in a Multi-Ethnic Asian Setting: Results from the Singapore-Malaysia Hospital-Based Breast Cancer Registry. The Breast, 20, S75-S80. https://doi.org/10.1016/j.breast.2011.01.015

[29] Butt, Z., Haider, S.F., Arif, S., et al. (2012) Breast Cancer Risk Factors: A Comparison between Pre-Menopausal and Post-Menopausal Women. Journal of the Pakistan Medical Association, 62, 120.

[30] Surakasula, A., Nagarjunapu, G.C. and Raghavaiah, K.V. (2014) A Comparative Study of Pre- and Post-Menopausal Breast Cancer: Risk Factors, Presentation, Characteristics and Management. Journal of Research in Pharmacy Practice, 3, 12. https://doi.org/10.4103/2279-042X.132704

[31] Edge, S.B. and Compton, C.C. (2010) AJCC Cancer Staging Handbook: From the AJCC Cancer Staging Manual. Vol. 2010, American Joint Committee on Cancer, Springer, New York, 718.

[32] Kariri, M., Jalambo, M.O., Kanou, B., et al. (2017) Risk Factors for Breast Cancer in Gaza Strip, Palestine: A Case-Control Study. Clinical Nutrition Research, 6, 161-171. https://doi.org/10.7762/cnr.2017.6.3.161

[33] Loman, N., Johannsson, O., Kristoffersson, U., et al. (2001) Family History of Breast and Ovarian Cancers and BRCA1 and BRCA2 Mutations in a Population-Based Series of Early-Onset Breast Cancer. Journal of the National Cancer Institute, 93, 1215-1223. https://doi.org/10.1093/jnci/93.16.1215

[34] Malone, K.E., Daling, J.R., Neal, C., et al. (2000) Frequency of BRCA1/BRCA2 Mutations in a Population-Based Sample of Young Breast Carcinoma Cases. Cancer, 88, 1393-1402. https://doi.org/10.1002/(SICI)1097-0142(20000315)88:6<1393::AID-CNCR17>3.0.C O;2-P

[35] Haber, G., Ahmed, N.U. and Pekovic, V. (2012) Family History of Cancer and Its Association with Breast Cancer Risk Perception and Repeat Mammography. American Journal of Public Health, 102, 2322-2329. https://doi.org/10.2105/AJPH.2012.300786

[36] Buxton, J.A., Bottorff, J.L., Balneaves, L.G., et al. (2003) Women's Perceptions of Breast Cancer Risk: Are They Accurate? Canadian Journal of Public Health, 94, 422-426.

[37] Caruso, A., Vigna, C., Marozzo, B., et al. (2009) Subjective versus Objective Risk in Genetic Counseling for Hereditary Breast and/or Ovarian Cancers. Journal of Experimental \& Clinical Cancer Research, 28, 157. https://doi.org/10.1186/1756-9966-28-157

[38] Silk, K.J., Bigbsy, E., Volkman, J., et al. (2006) Formative Research on Adolescent and Adult Perceptions of Risk Factors for Breast Cancer. Social Science \& Medicine, 63, 3124-3136. https://doi.org/10.1016/j.socscimed.2006.08.010

[39] Aljohani, S., Saib, I. and Noorelahi, M. (2016) Women's Performance of Breast Cancer Screening (Breast Self-Examination, Clinical Breast Exam and Mammography). Advances in Breast Cancer Research, 6, 16. https://doi.org/10.4236/abcr.2017.61002

[40] Katapodi, M.C., Lee, K.A., Facione, N.C., et al. (2004) Predictors of Perceived Breast Cancer Risk and the Relation between Perceived Risk and Breast Cancer Screening: A Meta-Analytic Review. Preventive Medicine, 38, 388-402. 
https://doi.org/10.1016/j.ypmed.2003.11.012

[41] Key, T.J., Verkasalo, P.K. and Banks, E. (2001) Epidemiology of Breast Cancer. The Lancet Oncology, 2, 133-140. https://doi.org/10.1016/S1470-2045(00)00254-0

[42] Land, C.E., Tokunaga, M., Koyama, K., et al. (2003) Incidence of Female Breast Cancer among Atomic Bomb Survivors, Hiroshima and Nagasaki, 1950-1990. Radiation Research, 160, 707-717. https://doi.org/10.1667/RR3082

[43] Gledo, I., Pranjic, N., Drljević, K., et al. (2012) Female Breast Cancer in Relation to Exposure to Medical Iatrogenic Diagnostic Radiation during Life. Contemporary Oncology, 16, 551-556.

[44] Cardis, E., Vrijheid, M., Blettner, M., et al. (2005) Risk of Cancer after Low Doses of Ionising Radiation: Retrospective Cohort Study in 15 Countries. BMJ, 331, 77. https://doi.org/10.1136/bmj.38499.599861.E0

[45] Ferro, R., Parvathaneni, A., Patel, S., et al. (2012) Pesticides and Breast Cancer. Advances in Breast Cancer Research, 1, 30. https://doi.org/10.4236/abcr.2012.13005

[46] Boyle, P., Boniol, M., Koechlin, A., et al. (2012) Diabetes and Breast Cancer Risk: A Meta-Analysis. British Journal of Cancer, 107, 1608.

https://doi.org/10.1038/bjc.2012.414

[47] Abdeen, H. (2006) Chronic Diseases in Palestine: The Rising Tide. Bridges, 2, 4-7.

[48] Lindgren, A., Pukkala, E., Tuomilehto, J., et al. (2007) Incidence of Breast Cancer among Postmenopausal, Hypertensive Women. International Journal of Cancer, 121, 641-644. https://doi.org/10.1002/ijc.22689

[49] Agnoli, C., Berrino, F., Abagnato, C.A., et al. (2010) Metabolic Syndrome and Postmenopausal Breast Cancer in the ORDET Cohort: A Nested Case-Control Study. Nutrition, Metabolism and Cardiovascular Diseases, 20, 41-48.

https://doi.org/10.1016/j.numecd.2009.02.006

[50] Bjørge, T., Lukanova, A., Jonsson, H., et al. (2010) Metabolic Syndrome and Breast Cancer in the Me-Can (Metabolic Syndrome and Cancer) Project. Cancer Epidemiology and Prevention Biomarkers, 19, 1737-1745.

https://doi.org/10.1158/1055-9965.EPI-10-0230

[51] Reeves, K.W., McLaughlin, V., Fredman, L., et al. (2012) Components of Metabolic Syndrome and Risk of Breast Cancer by Prognostic Features in the Study of Osteoporotic Fractures Cohort. Cancer Causes \& Control, 23, 1241-1251. https://doi.org/10.1007/s10552-012-0002-2

[52] Franceschi, S., la Vecchia, C., Negri, E., et al. (1990) Breast Cancer Risk and History of Selected Medical Conditions Linked with Female Hormones. European Journal of Cancer, 26, 781-785. https://doi.org/10.1016/0277-5379(90)90151-I

[53] La Vecchia, C., Negri, E., Franceschi, S., et al. (1997) Body Mass Index and Post-Menopausal Breast Cancer: An Age-Specific Analysis. British Journal of Cancer, 75, 441-444. https://doi.org/10.1038/bjc.1997.73

[54] Potischman, N., Swanson, C.A., Coates, R.J., et al. (1999) Intake of Food Groups and Associated Micronutrients in Relation to Risk of Early-Stage Breast Cancer. International Journal of Cancer, 82, 315-321. https://doi.org/10.1002/(SICI)1097-0215(19990730)82:3<315::AID-IJC1>3.0.CO;2$\underline{\mathrm{N}}$

[55] Bao, P.P., Shu, X.O., Zheng, Y., Cai, H., et al. (2012) Fruit, Vegetable, and Animal Food Intake and Breast Cancer Risk by Hormone Receptor Status. Nutrition and Cancer, 64, 806-819. https://doi.org/10.1080/01635581.2012.707277 